\title{
Homenaje a los profesores de la Cátedra de Medicina Legal de la Universidad de Chile
}

Luis Ciocca Ga ${ }^{a}$, Pamela Bórquez V ${ }^{b}$, Raúl Burgos Sc. A tribute to the professors of forensic
medicine at the University of Chile

The outstanding work of professors of the chair of Forensic Medicine at the School of Medicine of the University of Chile is highlighted. This chair is divided in three periods, that are paralell to the history of Chilean medicine. During these periods, the chair initiated, developed and fortified its teaching activities. The practice of forensic medicine was institutionalized and spread throughout the country. Its field of action and knowledge areas also increased (Rev Méd Chile 2008; 136: 666-70).

(Key words: Education, medical; Foren sic medicine; History of Medicine)

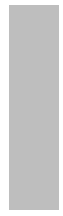

Recibido el 27 de noviembre, 2007. Aceptado el 10 de diciembre, 2007.

Departamento de Medicina Legal, Facultad de Medicina, Universidad de Chile. Santiago de Chile.

aOdontólogo Legista

bBecaria del Programa de Especialidad en Medicina Legal

cAlumno de la Carrera de Medicina

$E^{1}$ registro de los acontecimientos acaecidos en cualquier época y su interpretación, circunscritos a la actividad realizada por los participantes en ésta, conforman un cúmulo histórico que sienta las bases y el norte de quienes seguirán la misma senda en un futuro que se hace presente. La medicina chilena y su historia nos explican acontecimientos científicos, sociales, gremiales y culturales, desde que se fundara la primera ciudad en Chile hasta nuestros días. No escapa a ello la Medicina Legal, que ha sido impartida, desde la fundación de la Universidad de Chile, a todos los profesionales médicos formados por esta casa de

Correspondencia a: Dra. Pamela Bórquez V. Departamento de Medicina Legal, Facultad de Medicina, Universidad de Chile. Independencia № 1027, Independencia, Santiago. Fono: 9786003. Fax: 7776658. E mail: pamela.borquez@gmail.com estudios, para luego hacerse extensiva a las demás universidades del país como también a las profesiones de colaboración médica.

La dedicación de los médicos que fundaron y ejercieron esta disciplina auxiliar de la justicia, desde los primeros tiempos de la República hasta los actuales gobiernos, ha sido transversal a todos los sucesos históricos vividos por sus protagonistas; de este modo es posible destacar tres grandes períodos. El primero se caracteriza por el nacimiento y fortalecimiento de la cátedra dentro de la Universidad; el segundo, por la creación del Instituto Médico Legal y su desarrollo en la investigación de casos médico-legales como auxiliares de la justicia y el compromiso de la enseñanza de ésta. Por último, el tercer período se destaca por el crecimiento tecnológico del Servicio Médico Legal, la difusión de la Medicina Legal hacia la Odontología y otras profesiones de 
colaboración médica, su enseñanza en las universidades que están fuera de Santiago, la creación de servicios provinciales y el desarrollo de ésta hacia áreas tan importantes como la Responsabilidad Médica.

\section{Primer Período, de 1833 a 1901}

Época del romanticismo y de la emancipación (1810-1842)

El inicio formal de la Medicina Legal en Chile comienza con el Dr. Guillermo Blest Cunningham. De origen irlandés, al avecindarse en Chile, fundó, en 1833, la Escuela de Medicina de la Universidad de Chile, durante el gobierno del Presidente don José Joaquín Prieto Vial y la Rectoría de don Andrés Bello. El Dr. Blest fue profesor de varias asignaturas, entre ellas Medicina Legal entre 1833 y 1851, materia que probablemente había estudiado en su formación en Inglaterra. Posterior al Dr. Blest C., y ya en el gobierno de don Manuel Montt Torres, asume la enseñanza de la Medicina Legal entre 1851 y 1860, el Dr. Juan Miquel. De origen portugués, vino a Chile con el ejército español durante la guerra de la independencia. Ahí fue tomado prisionero y, tras ser liberado, ejerció la medicina así como la docencia en la naciente Escuela de Medicina, donde también impartió las cátedras de patología, clínica médica, terapéutica y toxicología. En esta época, sigue siendo Rector don Andrés Bello.

Época del positivismo y del liberalismo, 1842-1891

Ya establecida la cátedra de Medicina Legal, durante las Rectorías de don Andrés Bello y don Manuel Antonio Tocornal, el Dr. Vicente Padín del Valle fue docente de las asignaturas de Medicina Legal, entre 1860 y 1868, y Fisiología, siendo esta última su principal ocupación. Como Decano de la Facultad de Medicina, creó la primera Escuela de Flebotomía en 1863, antecesora de la Escuela Dental. El gobierno de la época estaba bajo el mando de don Manuel Montt Torres y luego de don José Joaquín Pérez. Su sucesor, el Dr. Pablo Zorrilla, después de haber sido profesor de las carreras de sangrador y flebótomo, fue designado para asumir las cátedras del Dr. Padín; pero, al revés de éste, se concentró en la enseñanza de la Medicina Legal entre 1868 y 1883, la cual ejerció durante las Rectorías de don Ignacio Domeyko y don Jorge Huneeus. Fue un gran docente y tuvo el mérito histórico de haber sido el primer profesor dedicado exclusivamente a la docencia de la Medicina Legal.

A la muerte del Dr. Zorrilla, el Dr. Federico Puga Borne fue designado profesor de Medicina Legal entre 1883 y 1897, siendo prácticamente el último académico del siglo XIX. Las Rectorías que supervisaron su trabajo docente fueron las de don Jorge Huneeus, José Ignacio Vergara, José Joaquín Aguirre, Diego Barros Arana y Diego San Cristóbal, en los agitados tiempos de los gobiernos de don Domingo Santa María, José Manuel Balmaceda, Jorge Montt Álvarez y Federico Errázuriz Echaurren. El Dr. Puga había ejercido la medicina en Valparaíso y se había interesado desde temprano por la Medicina Legal. Se ocupó de darle un cariz más práctico a la cátedra, haciendo que sus alumnos concurrieran a la morgue de la calle Teatinos. Entre sus publicaciones dejó un texto que llamó "Compendio de Medicina Legal", posiblemente el primero escrito por un chileno sobre estas materias. Pero sus labores no sólo eran del tipo académico, ya que fue un brillante político y diplomático, llegando a ser Ministro de Estado, Diputado y Senador, lo que finalmente motivó que renunciara a la cátedra que tan brillantemente ejerciera durante catorce años.

\section{Transición Del SIgLO XIX al XX}

Época de la Medicina Científica y el Parlamentarismo (1891-1927)

En el paso del siglo XIX al siglo XX, se encuentra al Dr. Gregorio Amunátegui Solar quien fue discípulo, en París, del ilustre médico legista francés Dr. Brouardel. Se ocupa de la disciplina entre los años 1897 y 1901, durante la Rectoría de don Diego San Cristóbal y Manuel Barros Borgoño, en el gobierno de don Federico Errázuriz Echaurren. Debido a que tenía otros intereses, al ser designado en Clínica, renunció a la cátedra de Medicina Legal. Su labor académica se coronó al ejercer como Decano de la Facultad de Medicina de la Universidad de Chile. 


\section{SEgundo PERÍodo de 1901 a 1959}

Quien sucediera al Dr. Amunátegui, los siguientes 27 años en la cátedra de Medicina Legal, es sin duda, el más grande de los maestros de la Medicina Legal chilena. Su paso por la cátedra marca un antes y un después en la Medicina Legal, ya que se proyectó en tiempo y espacio a las necesidades de esta nueva nación emergente que no llevaba no más de 100 años de independencia. El Dr. Carlos Ibar De la Sierra fue nombrado Profesor Titular de la Cátedra de Medicina Legal de la Facultad de Medicina de la Universidad de Chile, el día 10 de mayo de 1901. Al volver de Alemania, donde había viajado a perfeccionarse, se incorporó a la cátedra de Clínica Médica, cuyo profesor era el Dr. Wenceslao Díaz. Para el Dr. Ibar, la Clínica Médica era la disciplina por la cual se sentía atraído y en la que esperaba alcanzar su máximo desarmollo. Sin embargo, cuando llegó el momento, no fue designado para suceder al Profesor Díaz. Como compensación, en una suerte de mal menor, pero para bien de la disciplina, fue nombrado Profesor de la Cátedra de Medicina Legal, en 1901; permaneciendo en este cargo hasta 1928, entre los gobiemos de don Germán Riesco Errázuniz y el primero de don Carlos Ibáñez del Campo (Figura 1).

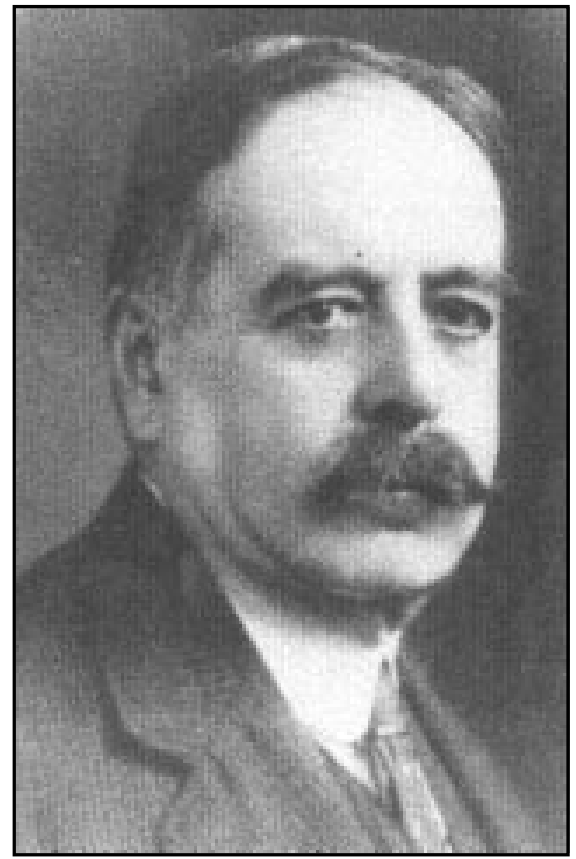

Figura 1. Dr. Carlos Ibar de la Sierra, Profesor de Medicina Legal de 1901 a 1928.
Estudioso y responsable como era, el Dr. Ibar hace primero un análisis de la situación de la enseñanza y el quehacer médico-legal, llegando a concluir que prácticamente había que hacerlo todo.

El Dr. Ibar, gestionó que el fisco adquiriera a la entonces llamada Beneficencia, terrenos ubicados al final de la Av. La Paz, a pocos metros del Cementerio General, donde se propuso levantar el primer Instituto de Medicina Legal que tuviera este país. Fue así como consiguió que el Ministerio del Interior dictara un Decreto Reglamentario, № 1049, del 30 de agosto de 1915, en el que se disponía que la Morgue de Santiago se trasladase desde su antiguo local de calle Teatinos a las nuevas instalaciones de Av. La Paz. En estos terrenos pronto se iniciaría la construcción del nuevo edificio del Instituto Médico Legal generado por el decreto mencionado. De este modo, la Morgue quedaba bajo la dirección del Profesor de Medicina Legal y éste sin derecho a percibir otra remuneración por el cargo. La continuidad entre las funciones docentes, periciales, asistenciales y de investigación se veían facilitadas por esta estructuración.

El Decreto № 1049 fue modificado el 17 de octubre de 1925 a través del Decreto Ley № 646 del Ministerio de Instrucción Pública, en el cual se estableció que los objetivos del Instituto Médico Legal eran servir a la justicia y a la enseñanza. Entretanto continuaban los trabajos de construcción del edificio de acuerdo a los planos conseguidos por el Dr. Ibar en Copenhague. Finalmente, se inauguró el Instituto el día 1 de octubre de 1926. Pero, no duró mucho la tranquilidad del profesor Ibar, pues en abril de 1928 se le pidió la renuncia al cargo sin darle ninguna razón. En ese momento se vivían días muy convulsionados desde el punto de vista político.

El Dr. Carlos Ibar, retirado ya, fallece el 3 de marzo de 1930. Algunos años después, en su honor y reconocimiento se le da su nombre al Instituto Médico Legal, que después de la salida del Dr. Ibar había dejado de pertenecer a la Universidad de Chile para ser un Servicio del Ministerio de Justicia.

Época contemporánea en la Medicina Chilena (1927 a la fecha)

Cuando se produjo la salida del Dr. Ibar, el Dr. Alberto Benítez Sanhueza era el legista más antiguo del Instituto Médico Legal, por lo que pasó a reemplazarlo en sus cargos, tanto como profesor de 
la cátedra como en la Dirección del Servicio. Ejenció sus funciones en el Departamento de Medicina Legal entre 1928 y 1931, bajo el gobiemo de don Carlos Ibáñez del Campo. El Dr. Benítez dejó escrito un Manual de Medicina Legal y entregó sus conocimientos a sus alumnos universitarios y a las Instituciones Policiales, que por esa época nacían. Fue el primer director de la Escuela de Investigaciones Policiales, fundada durante el segundo período de gobierno de don Arturo Alessandri Palma.

El Dr. Jaime Vidal Oltra, quien también fuera discípulo directo del Dr. Ibar, al suceder al Dr. Benítez Sanhueza, continuó la labor iniciada con el Profesor Ibar. Cuenta con ayudantes como los doctores Alfredo Vargas Baeza, Tomás Tobar Pinochet, Waldo Iturra, Augusto Veloso, Hernán Fuentes. Estuvo a cargo de la cátedra entre 1931 y 1959. Estudioso, se formó en la Escuela de Medicina de Panís, donde estudió en la Clínica de L'Hotel Dieu y en el Hôpital de la Pitié, donde hizo práctica de Laboratorio y Cardiología en el Hospital Broussais. También era un Médico Clínico que llegó a la Medicina Legal un poco en reemplazo de otros intereses (Figura 2).

En 1953 planteó la reorganización del Servicio que dirigía, considerando que se había producido un estancamiento y que no era sino un ente dedicado a la Tanatología, incluso con serias deficiencias técni-

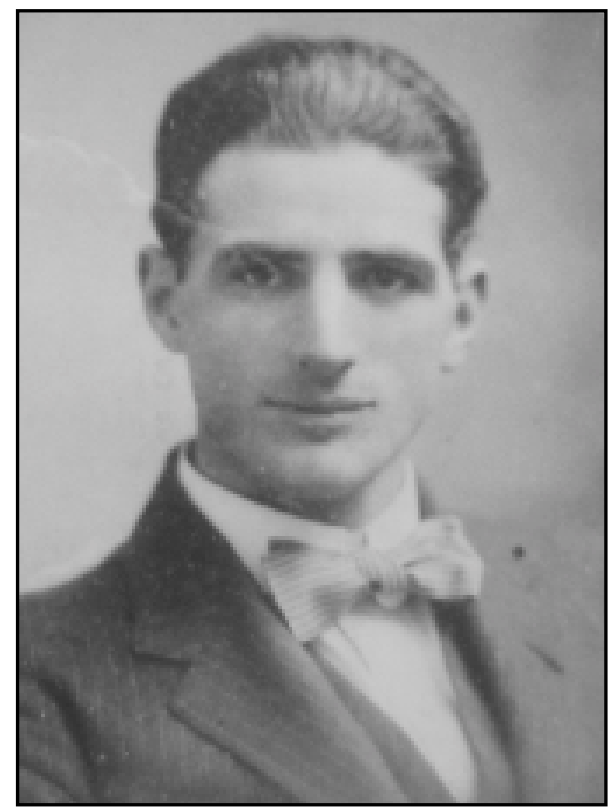

Figura 2. Dr. Jaime Vidal Oltra, Profesor de Medicina Legal de 1931 a 1959. cas. En esta reorganización consiguió importantes avances, pero su estado de salud lo obligó a dejar el cargo de director en el año 1955, continuando como profesor de la cátedra hasta 1959.

\section{TERCER PERÍODO, 1959 A 1997}

En este último período, el Dr. Alfredo Vargas Baeza desarrolló una labor extraordinaria en pro de la Medicina Legal chilena, ya que durante largo tiempo ocupó paralelamente los cargos de Director del Servicio Médico Legal, de profesor de la asignatura en la Universidad de Chile, y también de las Universidades Católica y de Concepción, las únicas formadoras de médicos del país en aquella época. Alcanzó a conocer en su juventud al Dr. Carlos Ibar, de quien también fue discípulo, siendo posteriormente ayudante del Dr. Vidal. Imbuido de las ideas de ambos, continuó trabajando por el desarrollo de la Medicina Legal, lo que sin duda consiguió, alcanzando un gran prestigio tanto nacional como internacional. El progreso se manifestó en ampliación y apoyo técnico a las distintas áreas del Servicio Médico Legal.

El Dr. Vargas Baeza tuvo un gran colaborador en el Dr. Tomas Tobar Pinochet, quien apoyó la iniciativa del Dr. Barrientos en la creación del Museo Médico Legal. El Dr. Tobar hizo acopio de una gran cantidad de piezas que se conservan todavía en perfectas condiciones (Figura 3).

Fue el Dr. Vargas Baeza quien inició la construcción de servicios provinciales, supervigiló constantemente la actividad del servicio, ejerció como perito tanatólogo y excelente docente.

Profesor invitado en diferentes universidades extranjeras, mantuvo contactos permanentes con diversos Servicios Médico-legales. Asistió y llevó el nombre de Chile a múltiples eventos intemacionales y en 1965 fue el motor de las Jomadas Internacionales de Medicina Legal, uno de los pocos eventos de esa envergadura celebrado en el país.

El 14 de abril de 1960 se dictó el Decreto con Fuerza de Ley № 196, a través del cual se reestructuró el Servicio, reguló la relación docente asistencial, organizó las diversas secciones, de las cuales aún se mantienen muchas.

El Dr. Vargas Baeza falleció en agosto de 1977, mientras se encontraba en ejercicio de todos sus cargos. Es sucedido por el Dr. Roberto Von Bennewitz. 


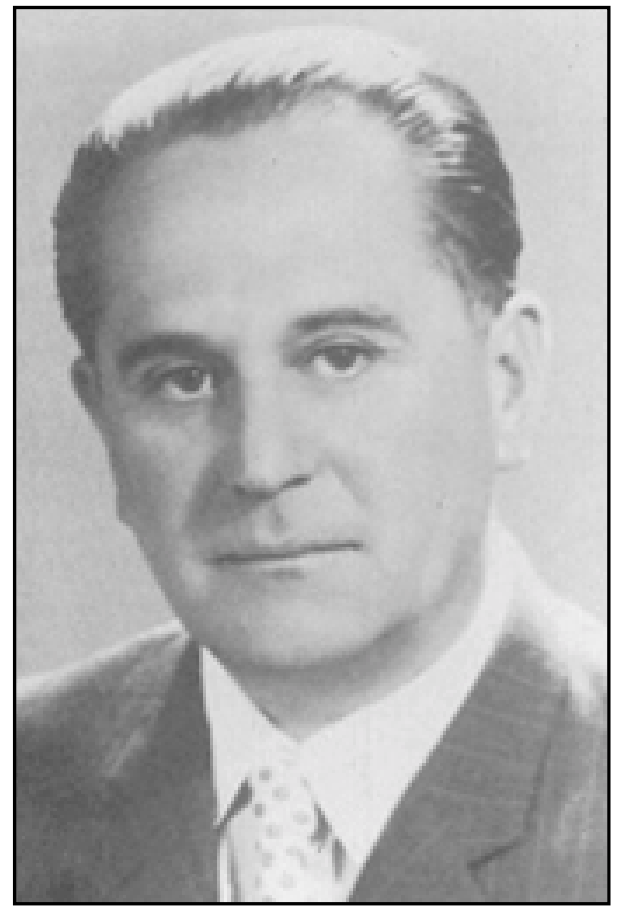

Figura 3. Dr. Alfredo Vargas Baeza, Profesor de Medicina Legal de 1959 a 1977.

El Dr. Roberto Von Bennewitz realiza su gestión en el Departamento durante 1977 y 1984. Coincide su período con el surgimiento de los primeros problemas médico-legales relacionados con la Responsabilidad Médica. La Medicina Legal comienza a difundirse hacia otras disciplinas como la Odontología, la Antropología, lo que la hace multidisciplinaria. Es así como se incorporan los primeros odontólogos al departamento. También se celebran convenios con las policías civil y

\section{REFERENCIAS}

1. Vicuña B. Médicos de Antaño. Buenos Aires, Argentina. Editorial Francisco De Aguirre. 1974.

2. OrRego A. Recuerdos de la Escuela. Buenos Aires, Argentina. Editorial Francisco De Aguirre. 1976.

3. CRUz Coke R. Historia de la Medicina Chilena. Santiago. Editorial Andrés Bello. 1995.

4. VIDALJ. Homenaje al Prof. Dr. Carlos Ibar de la Siema. Archivos Chilenos de Medicina Legal, Vol. I. 1959. uniformada a objeto de entregarles conocimientos médico-legales que les sean útiles en su labor.

Se realiza una vasta tarea de extensión del conocimiento médico-legal hacia los médicos y otros profesionales de la salud. Esto se consigue con cursos que se dictan prácticamente en todas las principales ciudades, además de la capital. Dentro de esta línea, en 1983 se realiza el primer curso de Medicina Legal dirigido a odontólogos lo que motiva también la fundación de la Sociedad de Odontología Legal de Chile. La enseñanza pierde su carácter meramente tanatológico y se enfatiza también aquellos aspectos del Derecho Médico y la Responsabilidad Profesional.

Por último, la docencia de la cátedra de Medicina Legal es continuada por el Dr. Alberto Teke Schlight, destacado médico criminalista. Fue jefe del Departamento de Medicina Criminalística de Investigaciones de Chile, Profesor de la Escuela de dicha institución entre 1967 y 1984 y Jefe de la Sección Tanatología del Servicio Médico Legal en 1978. En su período como Director del Departamento de Medicina Legal entre 1984 y 1997, se consolida la calidad multidisciplinaria de la Medicina Legal y del equipo docente de la Universidad. Se funda la Sociedad Chilena de Medicina Legal, Forense y Criminalística, la que preside. Se publican diversos trabajos como guías para la docencia, destacando el texto de "Medicina Legal". Se formó el Capítulo Chileno de la Asociación Mundial de Medicina Legal. Finalmente, se retiró el año 1997 de su cargo de director del Departamento manteniéndose actualmente muy activo en la docencia y la difusión de la disciplina.

Con esta revisión de la vida académica y logros, de los principales profesores de Medicina Legal de la Universidad de Chile, hemos querido rendir un homenaje a todos aquellos que se han dedicado a cimentar el camino de las futuras generaciones. 